STRUCTURAL SCIENCE CRYSTAL ENGINEERING MATERIALS

ISSN 2052-5206

Received 12 November 2020

Accepted 10 March 2021

Edited by A. Nangia, CSIR-National Chemical Laboratory, India

${ }^{1}$ Dedicated as a birthday tribute to Professor Wladek Minor, champion of quality and reproducibility in biostructural research, and a longtime friend.

Keywords: dual-conformation backbone; biogenic polyamine; biological potassium complex; subatomic resolution; Z-DNA.

PDB reference: Z-DNA complex with putrescine and potassium cations, 7 atg

Supporting information: this article has supporting information at journals.iucr.org/b

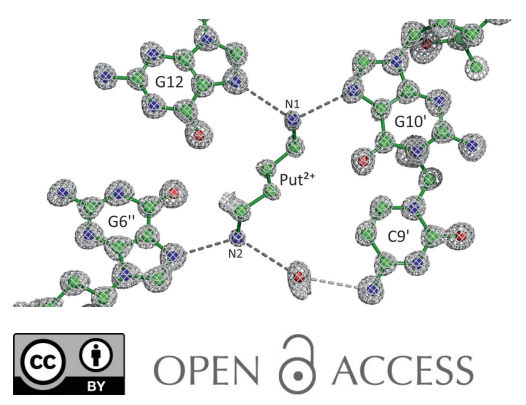

\section{Crystal structure of Z-DNA in complex with the polyamine putrescine and potassium cations at ultra-high resolution ${ }^{1}$}

\author{
Pawel Drozdzal, ${ }^{a}$ Miroslaw Gilski ${ }^{a, b}$ and Mariusz Jaskolski ${ }^{a, b *}$
}

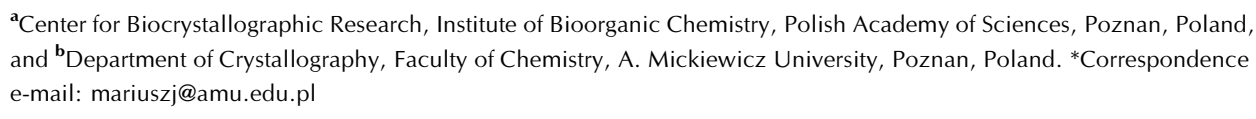

The X-ray crystal structure of the $\mathrm{d}(\mathrm{CGCGCG})_{2} /$ putrescine $(2+) / \mathrm{K}^{+}$complex has been determined at $0.60 \AA$ resolution. Stereochemical restraints were used only for the putrescinium dication, and 23 bonds and 18 angles of the Z-DNA nucleotides with dual conformation. The $\mathrm{N}$ atoms of the putrescine(2+) dication form three direct hydrogen bonds with the N7_G atoms of three different Z-DNA molecules, plus three water-mediated hydrogen bonds with cytosine, guanine and phosphate acceptors. A unique potassium cation was also unambiguously identified in the structure, albeit at a $\sim 0.5$ occupation site shared with a water molecule, providing the first example of such a complex with Z-DNA. The $\mathrm{K}^{+}$cation has coordination number of eight and an irregular coordination sphere, formed by four water molecules and four $\mathrm{O}$ atoms from two phosphate groups of the Z-DNA, including ligands present at fractional occupancy. The structural disorder of the Z-DNA duplex is manifested by the presence of alternate conformations along the DNA backbone. Comparison of the position and interactions of putrescine $(2+)$ in the present structure with other ultra-high-resolution structures of Z-DNA in complexes with $\mathrm{Mn}^{2+}$ and $\mathrm{Zn}^{2+}$ ions shows that the dicationic putrescinium moiety can effectively substitute these metal ions for stabilization of Z-type DNA duplexes. Furthermore, this comparison also suggests that the spermine(4+) tetracation has a higher affinity for Z-DNA than $\mathrm{K}^{+}$.

\section{Introduction}

Biogenic polyamine cations are essential for cell growth and differentiation, and their biochemical significance in a wide spectrum of physiological functions has been repeatedly reviewed (Gugliucci, 2004; Moinard et al., 2005; Larqué et al., 2007; Igarashi \& Kashiwagi, 2010; Jastrzab et al., 2016). Common compounds in this group include the diamines 1,3diaminopropane $(2+)\left(\mathrm{Dap}^{2+}\right)$, putrescine $(2+)\left(\mathrm{Put}^{2+}\right)$ and cadaverine $(2+)\left(\mathrm{Cad}^{2+}\right)$, the triamines spermidine $(3+)\left(\mathrm{Spd}^{3+}\right)$ and norspermidine(3+), and the tetramine spermine(4+) $\left(\mathrm{Spm}^{4+}\right) . \mathrm{Put}^{2+}$ and $\mathrm{Cad}^{2+}$ are the two most common biogenic diamines in bacteria. For example, at a concentration of 10 $30 \mathrm{~m} M$, putrescine is the predominant polyamine in Escherichia coli (Cohen, 1997). Polyamines $(n+)$ are involved in maintaining the cell-envelope integrity of bacteria. They are also crucial to the virulence phenotype of many bacterial pathogens (Shah \& Swiatlo, 2008; Di Martino et al., 2013). Moreover, polyamines $(n+)$ are important resources for viruses, which evolved mechanisms to maintain, enhance or manipulate polyamine $(n+)$ metabolism to support viral infection (Firpo \& Mounce, 2020). For instance, Mounce et al. (2017) showed the enhancement of viral polymerase activity 
by polyamines $(n+)$. Polyamines $(n+)$ are important factors stabilizing nucleic acids and stimulating their replication; only $\sim 7-10 \%$ of the total cell content exists as free polyamines $(n+)$, the vast majority remaining in association with nucleic acids (Gugliucci, 2004). It was also shown that both polyamines $(n+)$ and metal polycations (even at low concentration) can stimulate $\mathrm{B}$ to $\mathrm{Z}$ conversion of DNA and promote DNA condensation (Ohishi et al., 2008; Vijayanathan et al., 2001). Generally, both polyamines $(n+)$ and metal cations can interact with Z-DNA molecules in one of two different modes. Polyamine $(n+)$ cations are found to bind to the $\mathrm{O}$ atoms of different phosphate groups either directly or through watermediated bridges. In the second mode, the cations are coordinated simultaneously by the guanine bases from different helices and also by the $\mathrm{O}$ atoms of dual-conformation phosphate groups (Tomita et al., 1989; Gao et al., 1993).

Among all Z-DNA (Wang et al., 1979) complexes, the largest number of crystal structures in the Protein Data Bank (PDB; Berman et al., 2000) and Nucleic Acid Database (NDB; Narayanan et al., 2014) is available for complexes with magnesium and sperminium cations (Table 8 in Drozdzal, 2014). Z-DNA complexes with $\operatorname{Dap}^{2+}$ (PDB ID 2f8w), Spd $^{3+}$ (2elg and 293d), $N$-(2-aminoethyl)-1,4-diaminobutane (292d), thermospermine(4+) (336d), trientine(4+) (1dj6) and $N^{1}-\{2-$ [2-(2-aminoethylamino)ethylamino]ethyl\}ethane-1,2-diamine (2ie1) are also deposited in the PDB. Besides the $\mathrm{Mg}^{2+}$ cation, there are PDB structures of Z-DNA or Z-DNA-RNA (Gilski et al., 2016) duplexes with many other $\operatorname{metal}(n+)$ cations, including $\mathrm{Ba}^{2+}, \mathrm{Mn}^{2+}, \mathrm{Cu}^{2+}, \mathrm{Zn}^{2+}, \mathrm{Cr}^{3+}, \mathrm{Co}^{3+}$ and $\mathrm{Ru}^{3+}$. There is currently no structure, however, of a Z-DNA $/ \mathrm{K}^{+}$complex.

While the structure of the $\mathrm{Put}^{2+}$ cation has been thoroughly studied (Woo et al., 1979; Bratek-Wiewiorowska et al., 1986; Jaskólski, 1987; Pospieszna-Markiewicz et al., 2007), including the structures of phosphate salts (Jaskólski et al., 1986; Bartoszak \& Jaskólski, 1990) and a study at helium temperature (Jaskólski \& Olovsson, 1989), it is rather poorly characterized as a component of nucleic acid structures. The Put ${ }^{2+}$ dication was specified as a nucleic acid ligand only in nine structures deposited in the PDB, all of them corresponding to E. coli ribosome.

In this article, we describe a very-high-resolution crystal structure of Z-DNA with the sequence d(CGCGCG) $)_{2}$, which for the first time provides insight into the interaction of Z-DNA with $\mathrm{Put}^{2+}$ and $\mathrm{K}^{+}$cations. We also discuss the questions of competition and effectiveness of polyamine and metal cations in interactions with Z-DNA.

\section{Materials and methods}

\subsection{Oligonucleotide synthesis, purification and crystalliza-} tion

The methods of synthesis, deprotection and purification of the oligodeoxynucleotide have been described previously (Xia et al., 1998; Drozdzal et al., 2013). A $1.5 \mathrm{~m} M$ water solution of the DNA oligonucleotide with the self-complementary sequence $\mathrm{d}(\mathrm{CGCGCG})$ was heated at $338 \mathrm{~K}$ for $10 \mathrm{~min}$ and then
Table 1

Data collection and refinement statistics for $\mathrm{d}(\mathrm{CGCGCG})_{2} / \mathrm{Put}^{2+} / \mathrm{K}^{+}$.

\begin{tabular}{|c|c|}
\hline \multicolumn{2}{|l|}{ Data collection } \\
\hline Radiation source & P13, Petra III, DESY, Hamburg \\
\hline Wavelength $(\AA)$ & $0.6880,0.7293$ \\
\hline Temperature $(\mathrm{K})$ & 100 \\
\hline Space group & $P 2_{1} 2_{1} 2_{1}$ \\
\hline Cell dimensions $(\AA)$ & $a=17.97, b=31.02, c=43.86$ \\
\hline Resolution range $(\AA)$ & $25.33-0.60(0.61-0.60)^{a}$ \\
\hline Number of reflections & $107989^{b}$ \\
\hline Completeness native (\%) & $90.5(22.6)$ \\
\hline Redundancy & $3.7(1.2)$ \\
\hline$\langle I / \sigma I\rangle$ & $29.8(2.9)$ \\
\hline $\mathrm{CC}_{\perp}^{c}$ & $99.9(81.1)$ \\
\hline$R_{\text {merge }}{ }^{d}(\%)$ & $2.4(26.4)$ \\
\hline Wilson $B$-factor $\left(\AA^{2}\right)$ & 5.17 \\
\hline \multicolumn{2}{|l|}{ Refinement } \\
\hline Refinement program & SHELXL (Sheldrick, 2015) \\
\hline Resolution $(\AA)$ & $25.33-0.60$ \\
\hline No. of reflections in working set & $106103^{b}$ \\
\hline No. of reflections in test set & 1876 \\
\hline$R, R_{\text {free }}^{e}(\%)$ & $8.77,9.50$ \\
\hline $\begin{array}{l}\text { No. of atoms (nucleic acid, solvent, } \\
\text { polyamine, metal) }\end{array}$ & $240,123,6,1$ \\
\hline $\begin{array}{l}\langle B\rangle\left(\AA^{2}\right) \text { (nucleic acid chain } \mathrm{A}, \mathrm{B}, \\
\text { solvent, polyamine, metal) }\end{array}$ & $4.56,3.99,11.93,4.27,6.56$ \\
\hline $\begin{array}{l}\text { R.m.s. deviations from ideal bond } \\
\text { lengths }(\AA) \text {, angles }\left({ }^{\circ}\right)\end{array}$ & $0.010,1.56$ \\
\hline
\end{tabular}

Notes: (a) values in parentheses correspond to the last resolution shell; $(b)$ Bijvoet pairs separated; $(c)$ correlation between intensities from random half-sets as defined by Karplus \& Diederichs (2012); (d) $R_{\text {merge }}=\Sigma_{h} \Sigma_{j} \mid I_{j}-\left\langle I|| \Sigma_{h} \Sigma_{j} I_{j}\right.$, where $I_{j}$ is the intensity of observation $j$ of reflection $h ;(e) R=\Sigma_{h}|| F_{\mathrm{o}}|-| F_{\mathrm{c}}\left|/ \Sigma_{h}\right| F_{\mathrm{o}} \mid$ for all reflections, where $F_{\mathrm{o}}$ and $F_{\mathrm{c}}$ are the observed and calculated structure factors, respectively. $R_{\text {free }}$ was calculated analogously for the test reflections, which were randomly selected and excluded from the refinement.

annealed slowly to room temperature overnight. Single crystals of the $\mathrm{d}(\mathrm{CGCGCG})_{2} / \mathrm{Put}^{2+} / \mathrm{K}^{+}$complex were grown at $292 \mathrm{~K}$ by the hanging-drop vapour-diffusion method by mixing a $2 \mu \mathrm{l}$ nucleic acid solution and a $2 \mu \mathrm{l}$ precipitating solution consisting of $10 \%(v / v) \quad(+/-)$-2-methyl-2,4-pentanediol (MPD), $40 \mathrm{~m} M$ sodium cacodylate $\mathrm{pH}$ 6.0, $80 \mathrm{mM} \mathrm{KCl}, 12 \mathrm{mM}$ $\mathrm{NaCl}$ and $14 \mathrm{~m} M$ putrescinium dichloride. The drops were equilibrated against $0.5 \mathrm{ml} 80 \%(v / v)$ MPD. Crystals appeared within one week and grew to dimensions of $0.3 \mathrm{~mm} \times 0.1 \mathrm{~mm}$ $\times 0.1 \mathrm{~mm}$.

\subsection{X-ray data collection and processing}

$\mathrm{X}$-ray diffraction data for the Z-DNA $/ \mathrm{Put}^{2+} / \mathrm{K}^{+}$complex were collected to a resolution of $0.60 \AA$ on the EMBL beamline P13 (Cianci et al., 2017) of the PETRA III storage ring at DESY, Hamburg. The crystal was vitrified in a stream of cold nitrogen gas at $100 \mathrm{~K}$. The mother liquor served as the cryoprotectant solution. The diffraction data were collected in two passes using two wavelengths and the following crystal-todetector distances, oscillation ranges and numbers of images: $\lambda$ 0.6880 and $0.7293 \AA, 134$ and $134 \mathrm{~mm}, 0.5$ and $0.1^{\circ}$ and 360 and 1800 , respectively. The diffraction data were indexed, integrated and scaled using the $X D S$ package (Kabsch, 2010). The $\mathrm{X}$-ray data statistics are summarized in Table 1. 


\subsection{Structure solution and refinement}

The structure was solved by molecular replacement using PHASER (McCoy et al., 2007). The DNA part of the PDB structure 4hig, corresponding to our earlier model of the $\mathrm{d}(\mathrm{CGCGCG})_{2} / \mathrm{Spm}^{4+} / \mathrm{Mn}^{2+}$ complex (Drozdzal et al., 2013), served as a molecular probe. At the initial stages of the refinement, the model was refined using REFMAC5 (Murshudov et al., 2011) from the CCP4 program suite (Winn et al., 2011). The final anisotropic refinement was carried out with SHELXL (Sheldrick, 2015) using the full resolution of the diffraction data. The details of the SHELXL refinement were the same as described for our previous Z-DNA structures (Drozdzal et al., 2013, 2015), except for the use of the DISP instruction, which allows the definition of the dispersion and absorption coefficients of a particular element without having to use the full format of the SFAC instruction. The instruction OMIT was used to exclude ten of the most disagreeable reflections (error/e.s.d. $>10$ ) from the refinement. The model was validated using the NuCheck program (Feng et al., 1998) and the free $R$ test (Brünger, 1992), with 1876 reflections selected at random and set aside for $R_{\text {free }}$ calculations.

It should be noted that the atomic scattering factor for the $\mathrm{K}^{+}$site was declared on the SFAC instruction by specifying a neutral $\mathrm{K}$ atom. This small inaccuracy means assigning one excess electron per 18 actual electrons. This should have very little, if any, effect on the refinement, possibly underestimating the refinable occupancy of the $\mathrm{K}^{+}$cation by a factor of $18 / 19$.

At the wavelengths used in the diffraction experiments $(0.6880,0.7293 \AA)$, the imaginary component of the anom- alous scattering $\left(f^{\prime \prime}\right)$ of $\mathrm{K}$ and $\mathrm{P}$ atoms are, respectively, 0.236, 0.286 and 0.095, 0.104 electron units (Cromer, 1983). The anomalous signal is significant in the diffraction data set up to $\sim 0.9 \AA$ resolution, as illustrated by clear peaks at the $\mathrm{K}^{+}$ ion and $\mathrm{P}$ atoms in the anomalous electron-density map (Fig. 1).

At this resolution, no stereochemical restraints are necessary to supplement the experimental observations (Jaskolski, 2017). However, restraints may still be needed for some disordered or highly mobile fragments. In the present structure, restraints were applied only to the putrescinium dication and to bonds and angles (33 for the sugar and eight for the phosphodiester moieties) of dual-conformation Z-DNA fragments. The ideal geometry targets for $\mathrm{Put}^{2+}$ were taken from Pospieszna-Markiewicz et al. (2007). Conformation-dependent geometrical restraints on bond lengths (DFIX) and bond angles (DANG) for the polynucleotide chains were generated using the RestraintLib server (http://achesym.ibch.poznan.pl/ restraintlib/) as described by Kowiel et al. (2016, 2020) and Gilski et al. (2019). The CSD-derived conformation-dependent RestraintLib dictionary supersedes the classic nucleic acid restraints compiled by Parkinson et al. (1996). The final cycles of CGLS (conjugate-gradient least-squares) refinement converged with $R / R_{\text {free }}$ values of $8.88 / 9.50 \%$. The very last round of refinement, calculated with the test reflections included in the working set, converged with $R=8.77 \%$. In order to provide estimations of standard uncertainties in all individual refined parameters and of all derived geometrical parameters, at the final stage of the refinement, one cycle of full-matrix least-squares minimization was calculated. Model placement in the unit cell was standardized with the Achesym server (Kowiel et al., 2014).

(b)

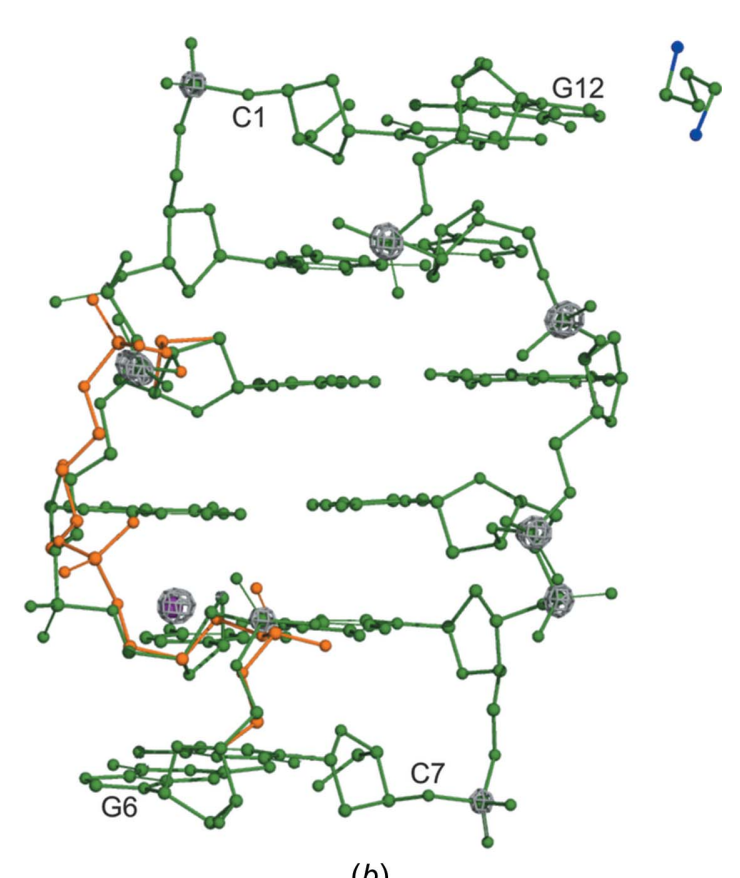

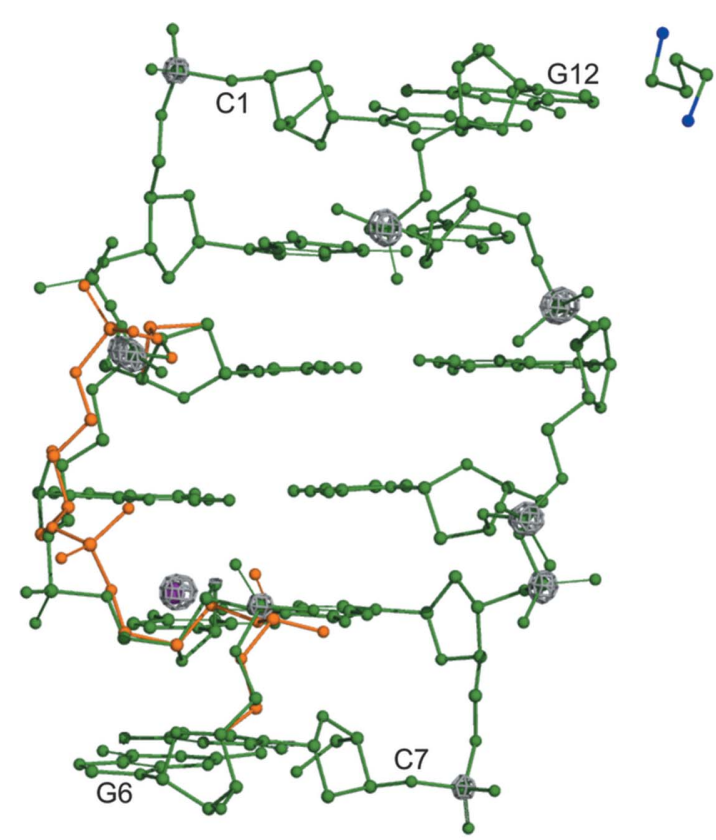

(a)

Figure 1

Stereoview of the $\mathrm{d}(\mathrm{CGCGCG})_{2} / \mathrm{Put}^{2+} / \mathrm{K}^{+}$complex with anomalous difference map (gray) peaks for the $\mathrm{K}^{+}$ion (purple sphere) and $\mathrm{P}$ atoms. The map is contoured at $3 \sigma$. Note the alternative conformations (I is green and II is orange) along the DNA chain. 
The final model includes all the $\mathrm{H}$ atoms of the oligonucleotides and $\mathrm{Put}^{2+}$ added as riding contributions to $F c$. The ammonium $-\mathrm{NH}_{3}{ }^{+}$groups of $\mathrm{Put}^{2+}$ were refined using the instruction AFIX 33. The rotatable $\mathrm{O}^{\prime} / 5^{\prime}-\mathrm{H}$ groups of the terminal sugars were refined using the instruction AFIX 87.

Figures presenting the model and electron density were prepared with PyMOL (DeLano, 2002). The Coot (Emsley et $a l ., 2010)$ program was used for visualization of the electrondensity maps and manual rebuilding of the atomic model. The program 3DNA (Lu \& Olson, 2003) was used to calculate the Z-DNA helical parameters. The pseudorotation parameters were calculated by the method of Jaskólski (1984) using the PseudoRotation server (http://www.cryst.ump.edu.pl/pseudorotation.php).

\subsection{Data deposition in public repositories}

Atomic coordinates and anisotropic ADPs, as well as the processed structure factors corresponding to the final model presented in this work, were deposited in the PDB with accession code 7atg. Raw X-ray diffraction images were deposited in the Integrated Resource for Reproducibility in Macromolecular Crystallography repository (proteindiffraction.org; Grabowski et al., 2016) with DOI https:// dx.doi.org/10.18430/m37atg.

\section{Results and discussion}

\subsection{Quality of the results}

The estimated standard uncertainties (e.s.u.) of the fully occupied DNA atomic positions in the structure are in the range $0.003-0.01 \AA$ for $\mathrm{C}$ atoms, $0.003-0.007 \AA$ for $\mathrm{N}$ atoms, 0.003-0.009 $\AA$ for O atoms and 0.001-0.002 $\AA$ for P atoms. The e.s.u. values for the full-occupancy covalent bonds are $\sim 0.005$, $\sim 0.004, \sim 0.004$ and $\sim 0.003 \AA$ for $\mathrm{C}-\mathrm{C}, \mathrm{C}-\mathrm{O}, \mathrm{C}-\mathrm{N}$ and $\mathrm{P}-\mathrm{O}$, respectively. The agreement with stereochemical standards (r.m.s.d.) is $0.010 \AA$ for bond lengths and $1.56^{\circ}$ for bond angles. These results are comparable in terms of accuracy and

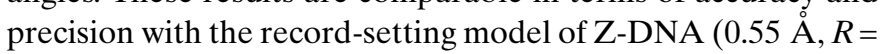
$7.77 \%$, PDB ID 3p4j) described by Brzezinski et al. (2011).

\subsection{Overall structure and helical parameters}

The overall structural parameters of the DNA moieties of the $\mathrm{d}(\mathrm{CGCGCG})_{2} / \mathrm{Put}^{2+} / \mathrm{K}^{+}$complex classify them within the Z-DNA family. The average helical twist $\Omega_{\mathrm{h}}$ (i.e. the angle of rotation about the helical axis that brings successive base pairs into coincidence) per CG/GC tandem of base pairs is $-60^{\circ}$. Other average base-pair and base-pair-step parameters are as follows: helical rise $4.37 \AA$, inclination $(\eta) 14.55^{\circ}$, tip $2.6^{\circ}$, tilt $0.44^{\circ}$, roll $-3.32^{\circ}$, shift $-0.02 \AA$, slide $2.77 \AA$ and rise $3.45 \AA$. Comparison of these helical parameters and base-pair geometries for the present and previously described Z-DNA complexes shows that they are within the range typical for Z-DNA duplexes (see supplementary Table S3 in Drozdzal et al., 2015).
The $5^{\prime}$-d(CGCGCG) nucleotides of chain $A$ in the asymmetric unit are numbered 1-6 and the complementary $3^{\prime}$ d(GCGCGC) nucleotides of chain B are numbered 12-7. There are only five internucleotide phosphodiester groups on each strand, which are numbered, respectively, P2-P6 and $\mathrm{P} 12-\mathrm{P} 8$. Alternative conformations are clearly visible in the electron-density map and are designated as I/II at the C3-G4, G4-C5 and C5-G6 internucleotide phosphate linkages, whose refined occupancies converged at $0.687(8) / 0.313(8)$, $0.710(4) / 0.290$ (4) and $0.650(12) / 0.350$ (12), respectively. The major (I) and minor (II) conformations of C3 have $\mathrm{C}^{\prime}$-endo and $\mathrm{C1}^{\prime}$-exo sugar puckers with pseudorotation $(\mathrm{P})$ angles of $162.0(7)$ and $138.2(6)^{\circ}$, respectively. Differences in sugar pucker are also observed at G4, where conformation I is $\mathrm{C}^{\prime}$ endo $\left[\mathrm{P}=20.2(7)^{\circ}\right]$ and conformation II is $\mathrm{C}^{\prime}$-exo $[\mathrm{P}=$ $\left.38(1)^{\circ}\right]$. Moreover, the $m F o-D F c$ electron-density maps indicate three additional discrete positions (peaks) for the phosphate atoms at the C3 $(6.7 \sigma), \mathrm{C} 9(6.0 \sigma)$ and C11 (6.7 $)$ nucleotides. However, additional phosphate groups placed at these peaks refined with an occupancy below 0.20 . Therefore, no atoms were modelled to interpret the peaks near the $\mathrm{P}_{-} \mathrm{C} 3$, P_C9 and P_C11 atoms. No alternative conformations were observed for the base moieties.

In the present complex, the sugars at the $3^{\prime}$-termini do not have the alternating $\mathrm{C} 2^{\prime}$-endo/ $\mathrm{C}^{\prime}$-endo pucker of the pyrimidine/purine nucleotides, as is typical for Z-DNA, but all assume the $\mathrm{C} 2^{\prime}$-endo conformation. The ZII conformation of the phosphate group can be assigned only to G4(I) with $\zeta=$ $64.6^{\circ}$ [ $\zeta$ is a backbone torsion angle defined as: $\mathrm{C3}^{\prime}-\mathrm{O}^{\prime}-$ $\left.\mathrm{P}(i+1)-\mathrm{O5}^{\prime}(i+1)\right]$. This ZII conformation is stabilized by a water-mediated OP2(I)_G4 ...Wat81 ...N2_G4 hydrogen bond.

\subsection{Coordination of the polyamine cation}

The entire putrescinium dication is clearly visible in the electron-density map (Fig. 2) despite its fractional occupancy, which converged on refinement at 0.378 (7). The putrescinium dication has a gauche ${ }^{-}$-trans-gauche $e^{+}$conformation, with the

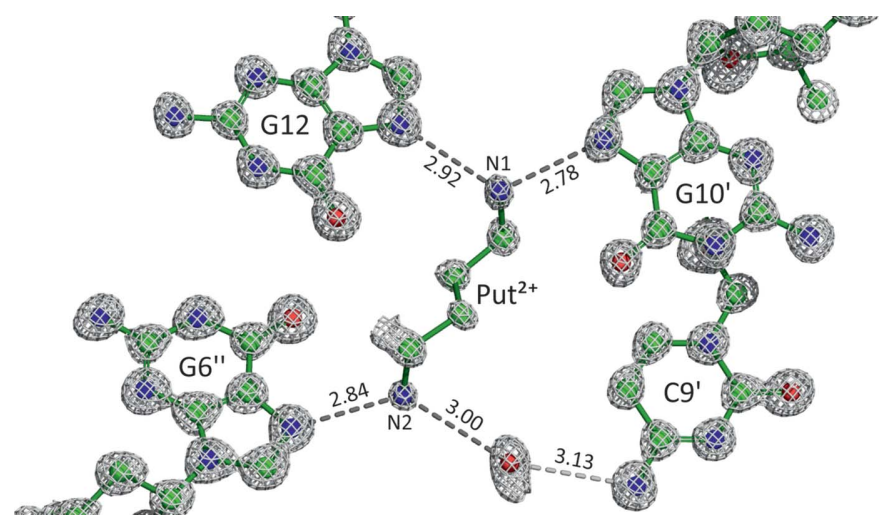

Figure 2

The putrescinium(2+) dication in the crystal structure of the $\mathrm{d}(\mathrm{CGCGCG})_{2} / \mathrm{Put}^{2+} / \mathrm{K}^{+}$complex. The $2 m F o-D F c$ map is contoured at the $1.6 \sigma$ level. Some water molecules have been omitted for clarity. Hydrogen bonds are marked by dashed lines, with $D \cdots A$ distances in $\AA$. 
Table 2

Coordination geometry $\left(\AA,^{\circ}\right)$ around the metal ion in the $\mathrm{d}(\mathrm{CGCGCG})_{2} / \mathrm{Put}^{2+} / \mathrm{K}^{+}$structure, with standard uncertainties in parentheses.

\begin{tabular}{|c|c|c|c|c|c|c|c|c|c|}
\hline & Distance & \multicolumn{8}{|c|}{ Angles } \\
\hline W110(I) & $2.612(22)$ & $80.3(6)$ & & & & & & & \\
\hline W110(II) & $2.831(39)$ & $72.3(10)$ & $12.4(8)$ & & & & & & \\
\hline OP1(I)_6 & $2.715(8)$ & $108.8(2)$ & $97.1(7)$ & $90.1(10)$ & $80.0(4)$ & & & & \\
\hline O5'(I)_6 & 3.047 (11) & $73.4(2)$ & $123.6(6)$ & $111(1)$ & $124.5(6)$ & $49.5(2)$ & & & \\
\hline W117 & $3.172(29)$ & 168.0 & $107.4(7)$ & 117 (1) & $29.8(6)$ & $79.9(3)$ & 108.3 & & \\
\hline OP1_9 $9^{\mathrm{iii}}$ & 3.185 (11) & $80.4(2)$ & $151.4(7)$ & $150.7(9)$ & $117.3(5)$ & $109.1(4)$ & 69.9 (3) & $89.0(5)$ & \\
\hline
\end{tabular}

Symmetry code: (iii) $-x+1, y-\frac{1}{2},-z+\frac{3}{2}$

following torsion angles: -72.5(7), $172.9(5)$ and $71.0(8)^{\circ}$. The $\mathrm{Put}^{2+}$ dication is involved in direct hydrogen-bond contacts only with the guanine N7 atoms of three DNA duplexes (Fig. 2). The N1 atom is anchored by the N7 atoms of two neighbouring DNA duplexes at the following distances: N1_Put ${ }^{2+} \ldots$ N7_G12 = 2.915 (6) $\AA$ and N1_Put ${ }^{2+} \ldots$ N7_G10 ${ }^{\mathrm{i}}=$ 2.755 (6) $\AA$ [symmetry code: (i) $x-\frac{1}{2},-y+\frac{3}{2},-z+1$ ]. The N2 atom is hydrogen bonded to N7_G6 $6^{\mathrm{ii}}$ at a distance of 2.839 (7) $\AA$ [symmetry code: (ii) $-x+\frac{3}{2},-y+1, z-\frac{1}{2}$ ]. The remaining three $\mathrm{N}-\mathrm{H}$ donors of the putrescinium dication form water-mediated hydrogen bonds with the O6_G10 N4_C9 ${ }^{\mathrm{i}}$ and OP1(I)_C5 ${ }^{\mathrm{ii}}$ atoms of two Z-DNA duplexes.

The electron-density maps indicate six water molecules in the vicinity of the $\mathrm{Put}^{2+}$ dication as alternative species populating the polyamine $(2+)$ site at complementary occupancy. It should be noted that there was a $\sim 20$-fold molar excess of $\mathrm{Put}^{2+}$ relative to the Z-DNA duplex in the crystallization mixture. The low occupancy of the $\mathrm{Put}^{2+}$ site is, therefore, not the result of insufficient supply of the ligand, but rather reflects the natural equilibrium of components required for growing those high-quality (from the point of view of diffraction quality) crystals.

There is very little literature information on putrescine $(2+)$ interactions with longer $\mathrm{d}(\mathrm{CG})_{n}$ sequences. Putrescine had no effect on the conformation of a plasmid (pDHg16) with a 23base pair $\mathrm{d}(\mathrm{GC})_{23}$ insert up to a $3 \mathrm{~m} M$ concentration (Thomas et al., 1991). Our results indicate that the putrescine $(2+)$ cation has preference for interactions with Z-DNA bases, which may explain why a $3 \mathrm{~m} M$ putrescine concentration was not sufficient for the B- to Z-DNA transition in the above plasmid. For longer $\mathrm{d}(\mathrm{GC})_{n}$ sequences in vitro, high concentrations of the putrescine $(2+)$ cation may be needed to lower the energetic cost of Z-DNA formation.

\subsection{Coordination of the $\mathrm{K}^{+}$cation}

The electron-density maps clearly revealed one metal coordination site with an occupancy of 0.49 (3), interpreted as potassium. Due to the partial occupation of the potassium cation, a complementary water molecule (Wat202) was also modeled in the $2 m F o-D F c$ map at this site with an occupancy of 0.34 (6). After the refinement of this model, the $R$ factor was reduced from 8.82 to $8.77 \%$. The refined distance between the $\mathrm{K}^{+}$and Wat202 sites is 0.195 (14) $\AA$.

The metal was unambiguously identified as potassium using the following pieces of evidence. The length of the $M-\mathrm{O}$ bonds supports the presence of $\mathrm{K}^{+}$rather than, for example, the presence of $\mathrm{Na}^{+}$at higher occupancy. In the anomalous difference map, its peak $(6.1 \sigma)$ had a height similar to that of a full-occupancy $\mathrm{P}$ atom $\left(\mathrm{P} \_11\right.$ at $\left.6.4 \sigma\right)$. The bond-valence method of Brese \& O'Keeffe (1991), which correlates bond valences with the identity of the metal atom, is a popular method in coordination chemistry, and is especially reliable at high resolution. The application of this method gives values of the valence $\left(V_{\mathrm{K}}\right)$ and bond-valence $\left(R_{\mathrm{KO}}\right)$ parameters of 1.18 , 1.09 and 2.07, 2.10 (the expected values for $\mathrm{K}^{+}$being $V_{\mathrm{K}}=1.00$ and $R_{\mathrm{KO}}=2.13$ ) for the coordination sphere including Wat110(I)/Wat110(II), respectively (vide infra). Also, the application of the CBVS method of Müller et al. (2003) confirmed the identity of the metal site as $\mathrm{K}^{+}$(calcium bondvalence sum $\mathrm{CBVS}=0.53$; according to the CBVS method, the reference value for $\mathrm{K}^{+}$is 0.64). Finally, the CheckMyMetal server (Zheng et al., 2014) also predicted potassium as the most likely cation at this site.

The $\mathrm{K}^{+}$cation is located between two Z-DNA phosphate groups. There are eight $\mathrm{O}$ atoms (four from Z-DNA backbone and four from water molecules) in the immediate vicinity (up

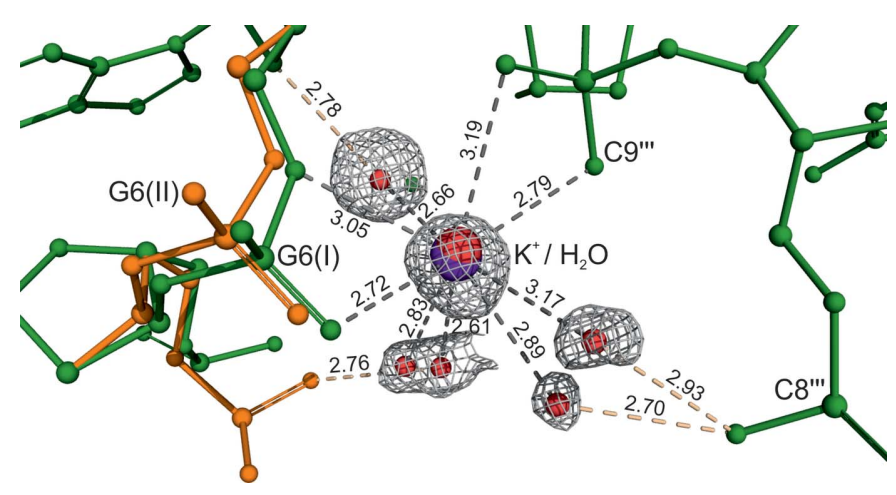

Figure 3

The coordination sphere of the hydrated complex of $\mathrm{K}^{+}$(purple) at G6. The $2 m F o-D F c$ map is contoured at $1.0 \sigma$. Coordination bonds are marked as gray dashed lines and hydrogen bonds are marked as orange dashed lines. Bond distances are in $\AA$. 
to a distance of $3.20 \AA$ ) of the $\mathrm{K}^{+}$cation (Table 2). However, one of the water molecules (Wat110) and one phosphate group (OP1_G6) interacting with the potassium cation are disordered and were modelled in two complementary positions. In the presence of the OP1(I) and $\mathrm{O}^{\prime}(\mathrm{I})$ atoms $(\mathrm{CN}=$ 8), the coordination sphere can be considered as highly distorted square antiprismatic or dodecahedral. The $\mathrm{K}^{+}$ion is coordinated simultaneously by OP1(I), O5'(I) from G6 and OP1, OP2 from C ${ }^{\text {iii }}$ [symmetry code: (iii) $-x+1, y-\frac{1}{2},-z+\frac{3}{2}$ ], as well as by four water sites, one of which (Wat110) has dual occupancy (Fig. 3 and Table 2). The $\mathrm{K}^{+}-\mathrm{O}$ bond distances are in the range $2.612(22)-3.185$ (11) $\AA$. The angles within the coordination sphere are irregular (Table 2).

\subsection{Hydration}

The asymmetric unit contains 123 water sites. All water molecules were refined anisotropically without positional restraints. There was no attempt to model the $\mathrm{H}$ atoms of the water molecules. While for some water molecules it might be possible to try to locate their $\mathrm{H}$ atoms from difference Fourier maps, such $\mathrm{H}$ atoms have notoriously very poor geometry, even in small-molecule structures, and in macromolecular structures the dubious gain from their inclusion in the model usually does not compensate the burden of their individual handling in the refinement (Jaskolski, 2017). Summation of all the water occupancies in the asymmetric unit gives a total water content of 93.15. The positions of many disordered water molecules are correlated with the alternate I/II conformations of the Z-DNA backbones. With respect to their occupancy parameters, those water molecules for which the occupancies converged on refinement to values close to unity $(>0.93)$ had their occupancy fixed at 1.0 ( 22 sites). Close pairs of sites for which the sum of their refined occupancies converged close to unity (36 pairs) had their combined occupancy constrained to 1.0. The remaining 65 sites had their occupancies refined freely to fractional values (all $\geq 0.20$ ). The common patterns of solvent structure, as noted previously for Z-DNA crystals (Drozdzal et al., 2013, 2015), such as water molecules between N2_G and phosphate $\mathrm{O}$ atoms, the spine of hydration (Chevrier et al., 1986), two water molecules hydrogen bonded to each O6_G group or the absence of water molecules hydrogen bonded to the N3_G atoms, are also observed in the present $\mathrm{Z}$-form structure.

\section{Discussion}

In this work, we have presented a new crystal structure of Z-DNA/polyamine $(n+)$, in complex with putrescine $(2+)$ and $\mathrm{K}^{+}$cations. It describes the first example of the interactions of putrescine(2+) with a DNA duplex. It is also the first case of Z-DNA crystallized in a complex with potassium ions. Moreover, the structure has the highest resolution and accuracy of the refined parameters among all DNA complexes with biogenic polyamines and/or metal cations deposited in the PDB.
Although the crystallization systems for all the Z-DNA complexes presented in our previous studies (Drozdzal et al., 2013,2015 ) always contained $\mathrm{KCl}$ at a concentration of 40 $80 \mathrm{mM}$ in the crystallization drop, the $\mathrm{K}^{+}$cation has been identified in the electron-density maps only in the present Z-DNA structure in complex with putrescine(2+). Comparison of the Z-DNA/Spm ${ }^{4+} / \mathrm{Mn}^{2+}$ and Z-DNA/ $\mathrm{Spm}^{4+} / \mathrm{Zn}^{2+}$ structures with the present Z-DNA/Put ${ }^{2+} / \mathrm{K}^{+}$complex in their common unit cell shows that the $\mathrm{N}^{+}$atoms of $\mathrm{Spm}^{4+}$ coincide almost exactly with the site of the $\mathrm{K}^{+}$cation. This may indicate that the $\mathrm{Spm}^{4+}$ cation has a higher affinity for Z-DNA binding than the $\mathrm{K}^{+}$cation and is preferentially selected when both are present in the crystallization buffer. It should also be stressed that the Z-DNA $/ \mathrm{K}^{+}$interaction has not been described in the literature so far in any crystallographic studies of Z-DNA structures. It is interesting to note that the general location of the $\mathrm{N}^{+}$and $\mathrm{N}^{+}$atoms of the putrescinium(2+) dication in the unit cell of the Z-DNA $/ \mathrm{Put}^{2+} / \mathrm{K}^{+}$complex is analogous to the positions of certain divalent metal cations, such as $\mathrm{Mn}^{2+}$ (PDB ID 4hig) or $\mathrm{Zn}^{2+}(1)$ and $\mathrm{Zn}^{2+}$ (2) (4hif) (Drozdzal et al., 2013) (Fig. 4). Therefore, $\mathrm{Put}^{2+}$, having the same net charge as $\mathrm{Mn}^{2+}$ or $\mathrm{Zn}^{2+}$, can effectively replace these cations in interactions with the Z-DNA duplex. It is also worth mentioning that putrescine $(2+)$ has a completely different interaction pattern than 1,3-diaminopropane(2+) $\left(\mathrm{Dap}^{2+}\right)$ and 1,5-pentanediamine(2+) [cadaverine(2+)]. The structure of Z-DNA with $\mathrm{Dap}^{2+}$ (PDB ID 2f8w) indicates that this one-carbon-linkshorter polyamine interacts only with the phosphate groups of the Z-DNA (Narayana et al., 2006). In a similar way, cadaverine(2+) (one carbon longer than $\mathrm{Put}^{2+}$ ) exhibits a preference for binding with Z-DNA phosphates only (Drozdzal et al., to be published).

The putrescinium dication has been characterized in several crystal structures of its salts and in some of them it has the gauche ${ }^{-}$-trans-gauche $e^{+}$conformation, while in the remaining cases it is all-trans (Woo et al., 1979; Jaskólski et al., 1986; Pospieszna-Markiewicz et al., 2007).

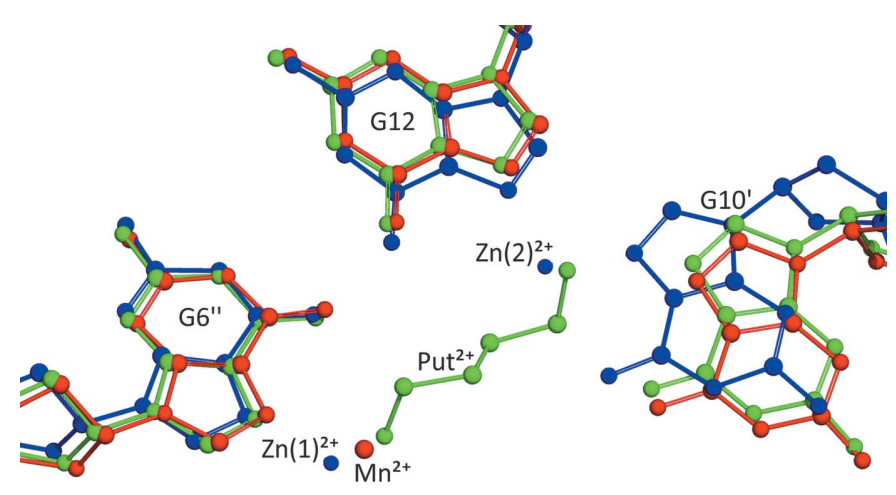

Figure 4

The d(CGCGCG) $)_{2} / \mathrm{Spm}^{4+} / \mathrm{Mn}^{2+}$ (red), d(CGCGCG) $)_{2} / \mathrm{Spm}^{4+} / \mathrm{Zn}^{2+}$ (blue) and $\mathrm{d}(\mathrm{CGCGCG})_{2} / \mathrm{Put}^{2+} / \mathrm{K}^{+}$(green) complexes in their common reference frame (unit cell). The interactions of the $\mathrm{Mn}^{2+}, \mathrm{Zn}^{2+}(1)$ and $\mathrm{Zn}^{2+}(2)$ ions and of the putrescinium(2+) N atoms with N7_G6, N7_G6 ${ }^{\mathrm{ii}}$ and N7_G10 ${ }^{\mathrm{i}}$ are indicated. A different location of $\mathrm{G} 10^{\mathrm{i}}$ in the complex with $\mathrm{Zn}^{2+}$ cations is also visible. 
Our study provides insight into the effectiveness and competition of polyamine and metal cations for interactions with Z-DNA, and confirms that even a simple diamine can adopt different conformations and consequently enter into a variety of interactions with biomacromolecular partners.

The partial occupancy of the $\mathrm{K}^{+}$ion confirms previous research findings suggesting that most observed monovalent cation sites in DNA crystals are partially occupied positions (Tereshko et al., 2001; Dong, 2003). These cations are mobile and easily exchange sites with water molecules. This result also agrees both with (i) the conclusion from molecular-dynamic simulations which suggested that the structures of monovalent counter-ions in DNA are dynamic (Young et al., 1997) and with (ii) the crystallographic studies on which the hybrid solvent model for the solvent structure around DNA is based and in which the solvent sites are occupied by water-cation hybrids (Shui et al., 1998).

\section{Acknowledgements}

We are grateful to Professor Ryszard Kierzek for the use of a DNA synthesizer. The synchrotron X-ray diffraction data were collected at beamline P13 operated by EMBL Hamburg at the PETRA III storage ring (DESY, Hamburg, Germany). We would like to thank Michele Cianci for assistance in using the beamline. This work was supported in part by a National Science Centre (NCN) grant.

\section{Funding information}

The following funding is acknowledged: National Science Centre (Poland) (grant No. 2013/10/M/NZ1/00251 to MJ).

\section{References}

Bartoszak, E. \& Jaskólski, M. (1990). Acta Cryst. C46, 2158-2160.

Berman, H. M., Westbrook, J., Feng, Z., Gilliland, G., Bhat, T. N., Weissig, H., Shindyalov, I. N. \& Bourne, P. E. (2000). Nucleic Acids Res. 28, 235-242.

Bratek-Wiewiorowska, M. D., Alejska, M., Perkowska, A., Wiewiorowski, M., Jaskolski, M. \& Cramer, F. (1986). Bull. Acad. Pol. Sci. Chim. 34, 229-249.

Brese, N. E. \& O'Keeffe, M. (1991). Acta Cryst. B47, 192-197.

Brünger, A. T. (1992). Nature, 355, 472-475.

Brzezinski, K., Brzuszkiewicz, A., Dauter, M., Kubicki, M., Jaskolski, M. \& Dauter, Z. (2011). Nucleic Acids Res. 39, 6238-6248.

Chevrier, B., Dock, A. C., Hartmann, B., Leng, M., Moras, D., Thuong, M. T. \& Westhof, E. (1986). J. Mol. Biol. 188, 707-719.

Cianci, M., Bourenkov, G., Pompidor, G., Karpics, I., Kallio, J., Bento, I., Roessle, M., Cipriani, F., Fiedler, S. \& Schneider, T. R. (2017). J. Synchrotron Rad. 24, 323-332.

Cohen, S. S. (1997). In A Guide to the Polyamines. New York: Oxford University Press Inc.

Coimbatore Narayanan, B., Westbrook, J., Ghosh, S., Petrov, A. I., Sweeney, B., Zirbel, C. L., Leontis, N. B. \& Berman, H. M. (2014). Nucleic Acids Res. 42, D114-D122.

Cromer, D. T. (1983). J. Appl. Cryst. 16, 437.

DeLano, W. L. (2002). The pyMOL Molecular Graphics System, Version 2.0. Schrödinger, LLC, New York, USA. https://pymol.org/2/. Di Martino, M. L., Campilongo, R., Casalino, M., Micheli, G., Colonna, B. \& Prosseda, G. (2013). Int. J. Med. Microbiol. 303, 484491.

Dong, X. (2003). Acta Cryst. D59, 1336-1338.
Drozdzal, P. (2014). PhD thesis. A. Mickiewicz University, Poznan, Poland. https://repozytorium.amu.edu.pl/handle/10593/10090.

Drozdzal, P., Gilski, M., Kierzek, R., Lomozik, L. \& Jaskolski, M. (2013). Acta Cryst. D69, 1180-1190.

Drozdzal, P., Gilski, M., Kierzek, R., Lomozik, L. \& Jaskolski, M. (2015). J. Biol. Inorg. Chem. 20, 595-602.

Emsley, P., Lohkamp, B., Scott, W. G. \& Cowtan, K. (2010). Acta Cryst. D66, 486-501.

Feng, Z., Westbrook, J. \& Berman, H. M. (1998). Report NDB-407. Rutgers University, New Brunswick, New Jersey, USA.

Firpo, M. R. \& Mounce, B. C. (2020). Biomolecules, 10, 628.

Gao, Y.-G., Sriram, M. \& Wang, A. H.-J. (1993). Nucleic Acids Res. 21, 4093-4101.

Gilski, M., Drozdzal, P., Kierzek, R. \& Jaskolski, M. (2016). Acta Cryst. D72, 211-223.

Gilski, M., Zhao, J., Kowiel, M., Brzezinski, D., Turner, D. H. \& Jaskolski, M. (2019). Acta Cryst. B75, 235-245.

Grabowski, M., Langner, K. M., Cymborowski, M., Porebski, P. J., Sroka, P., Zheng, H., Cooper, D. R., Zimmerman, M. D., Elsliger, M.-A., Burley, S. K. \& Minor, W. (2016). Acta Cryst. D72, 11811193.

Gugliucci, A. (2004). Clin. Chim. Acta, 344, 23-35.

Igarashi, K. \& Kashiwagi, K. (2010). Int. J. Biochem. Cell. Biol. 42, 39-51.

Jaskólski, M. (1984). Acta Cryst. A40, 364-366.

Jaskólski, M. (1987). Acta Cryst. C43, 1761-1763.

Jaskolski, M. (2017). In Protein Crystallography: Methods and Protocols, Vol. 1607, pp. 549-563 in the series Methods in Molecular Biology edited A. Wlodawer, Z. Dauter \& M. Jaskolski. Totowa, New Jersey: Springer - Humana Press.

Jaskólski, M., Alejska, M. \& Wiewiórowski, M. (1986). J. Crystallogr. Spectrosc. Res. 16, 31-39.

Jaskólski, M. \& Olovsson, I. (1989). Acta Cryst. B45, 78-85.

Jastrzab, R., Lomozik, L. \& Tylkowski, B. (2016). Phys. Sci. Rev. 1, 20160003.

Kabsch, W. (2010). Acta Cryst. D66, 125-132.

Karplus, P. A. \& Diederichs, K. (2012). Science, 336, 1030 1033.

Kowiel, M., Brzezinski, D., Gilski, M. \& Jaskolski, M. (2020). Nucleic Acids Res. 48, 962-973.

Kowiel, M., Brzezinski, D. \& Jaskolski, M. (2016). Nucleic Acids Res. 44, 8479-8489.

Kowiel, M., Jaskolski, M. \& Dauter, Z. (2014). Acta Cryst. D70, 32903298.

Larqué, E., Sabater-Molina, M. \& Zamora, S. (2007). Nutrition, 23, 87-95.

Lu, X. J. \& Olson, W. K. (2003). Nucleic Acids Res. 31, 51085121.

McCoy, A. J., Grosse-Kunstleve, R. W., Adams, P. D., Winn, M. D., Storoni, L. C. \& Read, R. J. (2007). J. Appl. Cryst. 40, 658674.

Moinard, C., Cynober, L. \& de Bandt, J.-P. (2005). Clin. Nutr. 24, 184 197.

Mounce, B. C., Olsen, M. E., Vignuzzi, M. \& Connor, J. H. (2017). Microbiol. Mol. Biol. Rev. 81, e00029-17.

Müller, P., Köpke, S. \& Sheldrick, G. M. (2003). Acta Cryst. D59, 3237.

Murshudov, G. N., Skubák, P., Lebedev, A. A., Pannu, N. S., Steiner, R. A., Nicholls, R. A., Winn, M. D., Long, F. \& Vagin, A. A. (2011). Acta Cryst. D67, 355-367.

Narayana, N., Shamala, N., Ganesh, K. N. \& Viswamitra, M. A. (2006). Biochemistry, 45, 1200-1211.

Ohishi, H., Odoko, M., Grzeskowiak, K., Hiyama, Y., Tsukamoto, K., Maezaki, N., Ishida, T., Tanaka, T., Okabe, N., Fukuyama, K., Zhou, D. Y. \& Nakatani, K. (2008). Biochem. Biophys. Res. Commun. 366, 275-280.

Parkinson, G., Vojtechovsky, J., Clowney, L., Brünger, A. T. \& Berman, H. M. (1996). Acta Cryst. D52, 57-64. 
Pospieszna-Markiewicz, I., Radecka-Paryzek, W. \& Kubicki, M. (2007). Acta Cryst. E63, o3650.

Shah, P. \& Swiatlo, E. (2008). Mol. Microbiol. 68, 4-16.

Sheldrick, G. M. (2015). Acta Cryst. C71, 3-8.

Shui, X., Sines, C., McFail-Isom, L., VanDerveer, D. \& Williams, L. (1998). Biochemistry, 37, 16877-16887.

Tereshko, V., Wilds, C., Minasov, G., Prakash, T. P., Maier, M., Howard, A., Wawrzak, Z., Manoharan, M. \& Egli, M. (2001). Nucleic Acids Res. 29, 1208-1215.

Thomas, T. J., Gunnia, U. B. \& Thomas, T. (1991). J. Biol. Chem. 266, 6137-6141.

Tomita, K., Hakoshima, T., Inubushi, K., Kunisawa, S., Ohishi, H., van der Marel, G. A., van Boom, J. H., Wang, A. H.-J. \& Rich, A. (1989). J. Mol. Graph. 7, 71-75.

Vijayanathan, V., Thomas, T., Shirahata, A. \& Thomas, T. J. (2001). Biochemistry, 40, 13644-13651.
Wang, A. H.-J., Quigley, G. J., Kolpak, F. J., Crawford, J. L., van Boom, J. H., van der Marel, G. \& Rich, A. (1979). Nature, 282, 680-686.

Winn, M. D., Ballard, C. C., Cowtan, K. D., Dodson, E. J., Emsley, P., Evans, P. R., Keegan, R. M., Krissinel, E. B., Leslie, A. G. W., McCoy, A., McNicholas, S. J., Murshudov, G. N., Pannu, N. S., Potterton, E. A., Powell, H. R., Read, R. J., Vagin, A. \& Wilson, K. S. (2011). Acta Cryst. D67, 235-242.

Woo, N. H., Seeman, N. C. \& Rich, A. (1979). Biopolymers, 18, 539552.

Xia, T., SantaLucia, J., Burkard, M. E., Kierzek, R., Schroeder, S. J., Jiao, X., Cox, C. \& Turner, D. H. (1998). Biochemistry, 37, 1471914735.

Young, M. A., Jayaram, B. \& Beveridge, D. L. (1997). J. Am. Chem. Soc. 119, 56-69.

Zheng, H., Chordia, M. D., Cooper, D. R., Chruszcz, M., Müller, P., Sheldrick, G. M. \& Minor, W. (2014). Nat. Protoc. 9, 156-170. 